\title{
BRAFV600E mutation and DNA ploidy determination in fine needle aspiration biopsy of papillary thyroid microcarcinoma in a Chinese population.
}

\author{
Liangyan Zhang ${ }^{1}$, Shengli Yan ${ }^{1 *}$, Guoliang Sui ${ }^{2}$, Yangang Wang ${ }^{1}$, Yubo Ren ${ }^{3}$, Chuanhong Li ${ }^{4}$ \\ ${ }^{1}$ Department of Endocrinology, the Affiliated Hospital of Qingdao University, Qingdao, PR China \\ ${ }^{2}$ Department of Endocrinology, Yantaishan Hospital, Yantai, PR China \\ ${ }^{3}$ Department of Pathology, Peking University International Hospital, Beijing, PR China \\ ${ }^{4}$ Department of Ultrasound, Yantaishan Hospital, Yantai, PR China
}

\begin{abstract}
This study aimed to evaluate the diagnostic value of BRAFV600E mutation and DNA ploidy determination for Papillary Thyroid Micro-Carcinoma (PTMC) through fine-needle aspiration. Clinical data, including histology, patient population statistics and clinical results were gathered. Patients' thyroid-micro-lesions were made into FNA-stained smears, DNA was extracted from the smears for different cytological diagnoses. BRAFV600E mutation was detected by Amplification Refractory Mutation System (ARMS). DNA ploidy determination of PTMC was detected by DNA quantitative analyser. All surgical cases were collected for histopathological diagnosis. BRAFV600E mutation was identified in 168 of $180(93.3 \%)$ of PTMC, 0/9 of thyroid follicular carcinomer and 0/1389 of benign thyroid nodules. 127/180 (70.5\%) patients got a definitive diagnosis of PTMC when only use cytology. 177/180 (98.3\%) patients got a definitive diagnosis of PTMC by a combination of cytology and BRAFV600E mutation analysis $(\mathbf{p}<0.05)$. DNA ploidy determination showed no abnormal on papillary thyroid microcarcinoma. BRAFV600E mutation could be only detected in Papillary Thyroid Carcinoma (PTC) patients and is the most common oncogenic alteration in PTC patients. Thus BRAFV600E mutation is a genetic marker of PTMC. BRAFV600E mutation analysis via fine-needle aspiration improved $27.8 \%$ of the diagnosis rate of PTMC when combined with cytology.
\end{abstract}

Keywords: Papillary thyroid carcinoma, BRAFV600E, DNA ploidy.

Accepted on March 30, 2017

\section{Introduction}

In worldwide, cancer is most important public health problem and in United States it is the second leading cause of death. In a human body, abnormal cells will grow in an abnormal way named as cancer and these cells are the cancer, malignant, or tumor cells. Normal body tissues are infiltrated by these cells. Cancer tissues are combined by many cancer and abnormal cells and these are identified by tissue name that the abnormal cells originated from such as breast cancer, lung cancer, colon cancer. Any organisms like human, animals, other can get cancer. From original mass of cells, the cancer cell break and passes through the blood and lymph systems, and lodge in other organs and this uncontrolled growth cycle has been repeated. By leaving an area these cancer cells grows in another body area and this process is named as metastatic spread or metastasis [1].

Thyroid cancer accounts for about $1 \%$ of all malignant tumors. It is the most common malignant tumor of thyroid, and is a kind of malignant tumor of thyroid epithelial cells. It is divided into many types include papillary thyroid carcinoma, thyroid follicular carcinoma, medullary thyroid carcinoma, anaplastic thyroid carcinoma. One of the most common is papillary thyroid, accounting for more than $90 \%$. The second is thyroid follicular carcinoma. Most common endocrine malignancy is the thyroid cancer accounts for $1.9 \%$ of all new malignant tumors by excluding skin cancer and in situ carcinomas are diagnosed annually in the United States. Considering $0.92 \%$ of cancers in men, $2.9 \%$ in women based on geographic area, age, and sex the annual incidence rates varied. From 1996 to 2000 in the United States, the age-adjusted annual incidence is 68 new cases per million, with a higher incidence in women (99/ million) than men (36/million). In United States with a female: male ratio close to $3: 1$, nearly 25,690 new cases of thyroid cancer is diagnosed as an annual report. In certain geographic areas for instance Hawaii with 119/million women and 45/ million men the incidence rates are highest possibly as a result of local environmental influences. Poland rates the lowest 
recorded as 14 per million women and four per million men. Under age of 15 , for children thyroid cancer is very rare. In this population, the annual U.S incidence is 2.2 per million girls and 0.9 per million boys. By the fifth through eighth decades, annual incidence of thyroid cancer increases as per age topping between 100 and 120 per million. Over a period of several decades in the United States and several other countries especially among women [2,3], the incidence of thyroid cancer has increased. Based on geographic area, patient population, and method of survey, occurrence rates vary for thyroid cancer. It has been reported as ranges from $0.03 \%$ to over $2 \%$ for the analysis rate. On 1000 consecutive routine autopsies, Mortenson et al. reported as a $2.8 \%$ prevalence rate of thyroid carcinoma. Occurrence of higher cancer is credited to the meticulous histological evaluation protocol. $61 \%$ of the cancers are originated from thyroid glands on the routine clinical assessment were apparently normal. Bisi et al. and Silverberg and Vidone proposed the similar prevalence rates $(2.3-2.7 \%)$. Reports of later studies stated the high prevalence rates which has been inclined by the highly selected inpatient populations studied but may not reflect the occurrence in the general population [4].

In papillary thyroid carcinoma, small foci measures $1 \mathrm{~cm}$ or less in diameter is classified as "papillary micro-carcinomas" and frequently occurred in autopsy material. Mostly papillary micro-carcinomas are measured ranges 4 and $7 \mathrm{~mm}$. These can be subdivided into "tiny" ranges 5-10 $\mathrm{mm}$ diameter and "minute" carcinomas has $<5 \mathrm{~mm}$ diameter. As encouraged by $\mathrm{Li}$, the "occult" carcinoma term has no pathological meaning. Meticulous sectioning of the thyroid detects the papillary micro-carcinomas ranging at 2-3 $\mathrm{mm}$ intervals and at each section, the detailed microscopic examination was presented. From Finland, report on highest prevalence rate of papillary thyroid micro-carcinoma ( $\leq 1 \mathrm{~cm}$ diameter) was presented with $33.7 \%$ of 101 cases harboring this finding. From Japan, rates over $20 \%$ have been reported. In Olmsted County, Minnesota the rate of papillary micro-carcinoma is lower which is $5.1 \%$. Clinically, the minute papillary carcinomas $(<5 \mathrm{~mm})$ are rarely detected and are believed to exhibit a relatively benign clinical course. The occasional reports of distant metastases such as pulmonary metastases that arise from minute papillary carcinomas $[5,6]$.

It is the most common malignancy of thyroid, occurs in approximately $90-95 \%$ of thyroid cancers. Its prevalence has been increasing in recent years because of the extensive use of ultrasound examination [7]. Thyroid nodules are detected by the Fine Needle Aspiration Biopsies (FNABS), about 10-30\% of the results are suspected FNA-diagnosis, especially for small thyroid nodule. The so-called microcarcinoma is papillary carcinoma with diameters less than or equal to $1 \mathrm{~cm}$. Postoperative pathological findings in patients with suspected malignancy were only $20-25 \%$ for thyroid cancer, while many patients received unnecessary surgery as seen in Yantai City of Shandong Province. How to correctly deal with these FNAB cannot determine the diagnosis of cases, is still facing the greatest difficulties and challenges. In recent years, novel molecular markers for thyroid cancers have been extensively investigated and proven to be clinically useful [8]. Whereas, the markers still have much space for improvement of the accuracy and precision of diagnostic and prognostic function. There are more new markers are needed to achieve these goals.

The $B R A F$ gene can activate the $B R A \mathrm{~F} / \mathrm{MEK} / \mathrm{ERK}$ pathway through the over activation and then occur cascade activation. It can result in abnormal cell proliferation and differentiation to lead to tumor formation. The predominant mutation in the $B R A F$ gene involves a thymidine to adenosine transversion at nucleotide 1,799 that accounts for greater than $90 \%$ of the observed mutations in $B R A F$ [9]. Silencing of multiple thyroidspecific iodine-metabolizing genes is related with the BRAFV600E. In synthesis of thyroid hormones, iodine is an important one. Inorganic iodine is transported into the thyroid cells and to follicular cells where it is oxidized and fused into thyroid hormones. In this process, decreased gene expression of several of the genes is associated by the BRAFV600E. BRAFV600E PTC and thyroid DNA methylation of the promoter regions was merged but not with RET/PTC rearrangement-induced PTC. The gene function was restored in thyroid cell model that carries BRAFV600E through the inhibition of MEK.

The mutation of $B R A F$ gene is characteristic for PTC. Some studies showed that this mutation is important for the onset and development of thyroid cancer, particularly papillary cancer. According to reports, the frequency of this mutation in PTC varies within the range of $29 \%$ to $92 \%$ in different population. The mutation frequency is closely related to location and race. Obvious differences were found between Asian and Western countries and Korean show the highest percent of BRAFrelated thyroid cancers in the present report in the world [10]. The association between $B R A F$ mutation and clinicpathological feathers remains controversial. So we further examined the frequency of this mutation in PTMC in a Chinese population and the preoperative diagnostic value of BRAFV600E mutation in the study.

\section{Literature Review}

$\mathrm{Li}$ et al. [11] established the prognostic value of the BRAFV600E mutation that results in poor clinical outcomes of papillary thyroid carcinoma. Aggressive clinical behaviors of PTMC have not been definitely established in individual studies and were associated with BRAFV600E. The relationship between BRAFV600E mutation and the clinicopathological features of PTMC was examined through this meta-analysis. Systematic search was conducted in PubMed, EMBASE, and the Cochrane library for relevant studies. From all studies the clinicopathological features of PTMC patients was reported with information available on BRAFV600E mutation status.

Lee et al. [12] concerned a role for Inter-Leukin 17 (IL17) in tumor development, uncharacterized the mechanisms of IL17 involvement. This work determined the Single Nucleotide Polymorphisms (SNPs) in IL17 and IL17R contributed for the development of PTC and assessed the relationship between 
IL17 and IL17R SNPs and the clinicopathological characteristics of PTC. Using direct sequencing in 94 patients with PTC and 260 patients without PTC (controls), eight SNPs located within the $I L 17 A, I L 17 R A$, and $I L 17 R B$ genes were genotyped. Commercially available software was analysed by the genetic data. The relationships between SNPs and the clinicopathological characteristics of PTC were examined by the statistical analyses.

Zhang et al. [13] made an investigation on association between a potentially functional polymorphism ( $\mathrm{rs} 153109,-964 \mathrm{~A}>\mathrm{G})$ in the promoter region of $I L-27$ gene and the risk of PTC in a Chinese population. Polymerase Chain Reaction-Restriction Fragment Length Polymorphism (PCR-RFLP) analysis was used to determine by the genotype of $I L-27-964 \mathrm{~A}>\mathrm{G}$ polymorphism. Enzyme-Linked Immuno-Sorbent Assay (ELISA) was used to determine the serum $I L-27 \mathrm{p} 28$ levels. The overall analysis stated that the significant difference was noticed in $I L-27-964 \mathrm{~A}>\mathrm{G}$ polymorphism between PTC patients and healthy controls. ELISA results demonstrate that serum $I L-27 \mathrm{p} 28$ levels were significantly decreased in PTC patients than with those in controls $(\mathrm{P}<0.05)$.

Wei et al. [14] observed an influence of selected Mature MicroRNAs on the risk of thyroid tumor. Seventeen mmSNPs were recognized after the systematic analysis in silico screening. In a Chinese population these are genotyped including 828 patients with PTC, 488 patients with benign thyroid tumor, and 1038 cancer-free controls. The association of SNP genotypes was evaluated by the analysis of multivariate logistic regression and alleles with the risk of developing PTC and BN. Three SNPs such as rs67106263 in mir-3144, GA vs. GG, OR=1.35, 1.09-1.68; rs4919510 in mir-608, CC vs. GG/GC, OR=0.76, 0.60-0.97; and rs79402775 in mir-933, AA vs. GG/GA, OR=1.76, 1.00-3.12 were related with PTC risk. To give increased PTC risk in a dose-dependent manner, it was observed the combined effect of unfavorable genotypes.

\section{Materials and Methods}

\section{FNAB and DNA preparation}

In more than one year period from 2014 to 2016, we recorded 756 patients who underwent thyroid colour ultrasonic examination and find 1578 thyroid nodules of diameter lessthan $1 \mathrm{~cm}$ at Yantai City of Shandong Province in China. This is a seaside area with low water iodine levels. We obtained 1578 FNAB specimens preoperatively from these thyroid nodules. Histopathological data were obtained from the case histories of these surgical patients. Institutional review board of Yantaishan Hospital (NO.1261) authorized this study. All participants provided written informed consent. FNAB cells coated on the carrier plate, hematoxylin-eosin stain and cytological diagnosis. Subsequently, $1 \mathrm{ml}$ saline was used to flush the remaining cells in the needle tips. Then freeze the washing liquid to below 80 degrees below zero for detection. The separation and extraction of genomic DNA from FNAB specimens is digested with proteinase $\mathrm{K}$, extracted with phenol-chloroform and precipitated with ethanol [15]

\section{Identification of BRAF V600E mutation}

A human BRAFV600E Detection Kit (YuanQi, Shanghai, China) based on Amplification Refractory Mutation System (ARMS) was utilized for detection of BRAF mutation. Each run contains a negative control and a positive control. An internal reference control was performed to ensure the presence of the DNA extracted from the tumor cells. The PCR was performed as follows: $95^{\circ} \mathrm{C}$ for $5 \mathrm{~min}$; two cycles of $95^{\circ} \mathrm{C} / 60 \mathrm{~s}$, $60^{\circ} \mathrm{C} / 60 \mathrm{~s}, 72^{\circ} \mathrm{C} / 60 \mathrm{~s}$; two cycles of $95^{\circ} \mathrm{C} / 60 \mathrm{~s}, 60^{\circ} \mathrm{C} / 60 \mathrm{~s}$, $58^{\circ} \mathrm{C} / 60 \mathrm{~s}, 72^{\circ} \mathrm{C} / 60 \mathrm{~s} ; 35$ cycles of $95^{\circ} \mathrm{C} / 60 \mathrm{~s}, 56^{\circ} \mathrm{C} / 60 \mathrm{~s}$, $72^{\circ} \mathrm{C} / 60 \mathrm{~s}$ and extension step is $72^{\circ} \mathrm{C}$ for $5 \mathrm{~min}$.

\section{DNA ploidy determination}

To determine the cumulative incidence of tumor recurrence, progression-free survival and overall survival DNA ploidy profile of the tumor plays an important prognostic factor and to identify a subgroup of cancer patients in need of adjuvant therapy. DNA ploidy measurements are used in human tumor diagnosis. The content of an abnormal DNA is a useful adjunct in the assessment, cytological diagnosis, and their potential to predict the disease outcome of human tumors such as Breast, Ovary, Endometrium, Prostate, Gastric, Kidney and Thyroid amongst others. The DNA content in cellular is difference between polyploidy or heteroploid tumor and normal cell. It depends on the degree of malignancy. The malignant thyroid carcinoma is in low degree. So the difference of the DNA content between malignant thyroid carcinoma and normal cell is small.

Importance of DNA ploidy was established by the analysis of flow cytometry and image to assess the biological activity of tumor and the prognostic difference between diploid and nondiploid tumors. To identify aneuploid subpopulations, the image cytometry is considered than flow cytometry that depends on reported findings on DNA ploidy. Image cytometry facilitates eliminates or discards the cut nuclei and duplets from the analysis and grouped into different clusters and so the reference cells do not interfere in DNA ploidy analysis of tumor leads to more accurate results. A Needle aspiration cell smeared on carrier film, 95\% alcohol fixation, feulgen stain and cell DNA quantitative analyser for detection.

\section{Statistical analysis}

We applied descriptive statistics to summary clinicopathological information and analyse categorical variables using the chi-square test. Statistical analysis was conducted using SPSS 17.0 software (SPSS Inc., California, USA). The calculation didn't contain the missing data and $\mathrm{p}<0.05$ was considered to indicate statistically significant.

\section{Results}

The frequency of BRAF V600E mutation in PTMC: BRAFV600E mutation was identified in 168 of $180(93.3 \%)$ 
PTMC, 0/9 of thyroid follicular carcinomer and 0/1389 benign thyroid nodules (Table 1). Histological diagnosis was obtained from postoperative results. The diagnostic value of BRAF V600E mutation in FNAB of PTMC: We observed that $127 / 180(70.5 \%)$ patients were able to get a clear diagnosis of PTMC only by the method of cytology (postoperative pathology confirmed). $177 / 180$ (98.3\%) patients were able to be appointed to a clear diagnosis of PTMC by BRAFV600E mutation united with cytology $(p<0.05$, Table 2$)$. After the detection of molecular detection, the BRAFV600E mutation results were $50 / 64(78.1 \%)$ cases with a cytology of unsatisfactory for PTMC and 118/127 (92.9\%) with definitive diagnosis of PTMC (Table 3). Consequently, the addition of $B R A F$ mutation detection can increase $27.8 \%$ of the diagnosis rate of PTMC compared to cytology alone.

Table 1. The result of histological, cytological diagnosis, positive $B R A F$ and BRAF mutation detection united with cytology.

\begin{tabular}{llll}
\hline Histological & Cytological & Positive BRAF & BRAF united with cytology \\
\hline PTMC (180) & $127(70.5 \%)$ & $168(93.3 \%)$ & $177(98.3 \%)$ \\
\hline Benign (1389) & 1389 & 0 & 1389 \\
\hline
\end{tabular}

Table 2. The result of BRAF mutation detection.

\begin{tabular}{lll}
\hline Categories & Positive BRAF & Negative BRAF \\
\hline Suspicious cytology of PTMC (64) & $50(78.1 \%)$ & $14(21.9 \%)$ \\
\hline Definitive cytology of PTMC (127) & $118(92.9 \%)$ & $9(7.1 \%)$ \\
\hline Definitive histology of PTMC (180) & $168(93.3 \%)$ & $12(6.7 \%)$ \\
\hline
\end{tabular}

Table 3. The comparison of cytological and BRAF united with cytology (chi-square test).

\begin{tabular}{lllll}
\hline & Positive BRAF & $\begin{array}{l}\text { Negative } \\
\text { BRAF }\end{array}$ & $\mathbf{X}^{2}$ & $\mathbf{p}$ \\
\hline BRAF united with cytology & $177(98.3 \%)$ & $3(1.7 \%)$ & 52.867 & $<0.05$ \\
\hline Cytological & $127(70.5 \%)$ & $53(29.5 \%)$ & & \\
\hline
\end{tabular}

\section{DNA ploidy determination in FNAB of PTMC}

DNA ploidy determination has no abnormal on papillary thyroid microcarcinoma $(0 / 180)$ on FNAB samples. So DNA ploidy determination is no diagnosis value for papillary thyroid microcarcinoma preoperative.

\section{Discussion}

Thyroid nodules are very common, ranging from 3\%-7\% by palpation and $20 \%-76 \%$ by ultrasound in the general population [16]. In the recent ten years, more and more thyroid micro-nodules are discovered by ultrasound. The proportion of papillary thyroid microcarcinoma in surgery is very high. Although most of the thyroid nodules are benign, differentiating malignancy from benign nodules is still dilemma for clinicians [17]. In 2009, Horvath et al. established a Thyroid Imaging Reporting and Data System (TIRADS) to interpret the thyroid nodules [18]. There is still a considerable part of the ultrasound suspicious of malignancy or cannot be identified in the thyroid nodules. Us-guided FNAB is present the most significant and reliable approach for the preoperative evaluation of thyroid nodules. But about $10-30 \%$ of the FNAB of thyroid nodules are detected as "indeterminate". Recently, researchers attempted to seek molecular markers that may enhance the diagnosis of FNAB. The most frequent genetic alternate in PTC is the BRAFV600E mutation. It accelerates PTC oncogenesis through specially activating the mitogenactivated protein kinase pathway and some other mechanisms [19]. Studies have shown that BRAFV600E mutation is only present in PTC and PTC-derived anaplastic thyroid cancers. It is a breakthrough in the molecular detection of thyroid carcinoma for the past few years [20,21]. In our study, all the nodules with BRAFV600E mutation were confirmed to be PTC. The detection method we used in this study is ARMS [22], and it is more sensitive and specific than the traditional method like DNA direct sequencing.

In our study, the mutation rate of the BRAFV600E in the Chinese population is $93.3 \%$, close to that in the Korea, but higher than the average level which was reported in the documents. Area and ethnic variations may be the main contributors to this difference [23]. It is very high. While PTC is a low potential malignancy, BRAFV600E mutation maybe a pathogenic factor of PTC. The positive maybe not related to the prognosis. The association between BRAF mutation and clinicpathological feathers remains controversial [24]. We need a large number of clinical evidence to support this view. For this study, we also assessed the effectiveness of FNAB and BRAFV600E mutation detection in differentiating thyroid nodules in clinical practice. The sensitivity and specifity of FNA-BRAFV600E detection are much better than those of cytology and ultrasound [25]. It is a powerful adjunct in evaluating PTC in Chinese population. The accuracy of cytology diagnosis depends on pathologists' experience and sample' quantity while BRAFV600E analysis only needs appropriate sample and standardized test [26,27]. 98.3\% patients could be considered a definitive detection of PTC when BRAFV600E mutation analysis united with cytology in our study [28].

Highly malignant tumor can be seen in the DNA times of the abnormal content of the tumor. PTC is a low grade malignancy. So PTC has normal DNA ploidy determination in both cytological and histological specimens. DNA ploidy determination is no diagnosis value for papillary thyroid microcarcinoma preoperative. In conclusion, the combination of cytology and BRAFV600E mutation analysis is reliable and efficient method for diagnosing PTC, particular for PTMC. Further studies will still need to verify our results.

\section{References}

1. Siegel RL, Miller KD, Jemal A. Cancer statistics, 2016. Cancer J Clin 2016; 66: 7-30.

2. Figge JJ. Epidemiology of thyroid cancer. Thyroid Cancer Springer New York 2016; 9-15. 


\section{microcarcinoma in a Chinese population}

3. La Vecchia C, Malvezzi M, Bosetti C, Garavello W, Bertuccio P. Thyroid cancer mortality and incidence: a global overview. Int J Cancer 2015; 136: 2187-2195.

4. Francis GL, Waguespack SG, Bauer AJ, Angelos P, Benvenga S, Cerutti JM, Parisi MT. Management guidelines for children with thyroid nodules and differentiated thyroid cancer: the American Thyroid Association guidelines task force on pediatric thyroid cancer. Thyroid 2015; 25: 716-759.

5. Tsuda T, Tokinobu A, Yamamoto E, Suzuki E. Thyroid cancer detection by ultrasound among residents ages 18 years and younger in Fukushima, Japan: 2011 to 2014. Epidemiol Cambridge Mass 2016; 27: 316.

6. Haugen BR, Alexander EK, Bible KC, Doherty GM, Mandel SJ, Nikiforov YE, Schuff KG. 2015 American Thyroid Association management guidelines for adult patients with thyroid nodules and differentiated thyroid cancer: the American Thyroid Association guidelines task force on thyroid nodules and differentiated thyroid cancer. Thyroid 2016; 26: 1-133.

7. Raposo L, Morais S, Oliveira MJ, Marques AP, Jose Bento M. Trends in thyroid cancer incidence and mortality in Portugal. Eur J Cancer Prev 2017; 26: 135-143.

8. Afkhami M, Karunamurthy A, Chiosea S, Nikiforova MN, Seethala R, Nikiforov YE, Coyne C. Histopathologic and clinical characterization of thyroid tumors carrying the BRAF (K601E) mutation. Thyroid 2016; 26: 242-247.

9. Cantwell-Dorris ER, Oleary JJ, Sheils OM. BRAFV600E: implications for carcinogenesis and molecular therapy. Mol Cancer Ther 2011; 10: 385-394.

10. Rowe LR, Bentz BG, Bentz JS. Utility of BRAF V600E mutation detection in cytologically indeterminate thyroid nodules. Cyto J 2006; 3: 10.

11. Li F, Chen G, Sheng C, Gusdon AM, Huang Y, Lv Z, Qu S. BRAFV600E mutation in papillary thyroid microcarcinoma: a meta-analysis. Endocr Cancer 2015; 22: 159-168.

12. Lee YC, Chung JH, Kim SK, Rhee SY, Chon S, Oh SJ, Eun YG. Association between interleukin 17/interleukin 17 receptor gene polymorphisms and papillary thyroid cancer in Korean population. Cytokine 2015; 71: 283-288.

13. Zhang S, Gao X, Wang Y, Jia J, Zhang Q, Ji Z. Interleukin 27-964A $>\mathrm{G}$ genetic polymorphism and serum IL-27p28 levels in Chinese patients with papillary thyroid cancer. Tumor Biol 2015; 36: 8207-8211.

14. Wei WJ, Wang YL, Li DS, Wang Y, Wang XF, Zhu YX, Wang JC. Association study of single nucleotide polymorphisms in mature microRNAs and the risk of thyroid tumor in a Chinese population. Endocrine 2015; 49: 436-444.

15. Popoveniuc G, Jonklaas J. Thyroid nodules. Med Clin North Am 2012; 96: 329-349.
16. Bomeli SR, Lebeau SO, Ferris RL. Evaluation of a thyroid nodule. Otolaryngol Clin North Am 2010; 43: 229-238.

17. Wei X, Li Y, Zhang S, Gao M. Thyroid imaging reporting and data system (TI-RADS) in the diagnostic value of thyroid nodules: a systematic review. Tumour Biol 2014; 35: 6769-6776.

18. Xing M. BRAF mutation in thyroid cancer. Endocr Relat Cancer 2005; 12: 245-262.

19. Xing M, Haugen BR, Schlumberger M. Progress in molecular-based management of differentiated thyroid cancer. Lancet 2013; 381: 1058-1069.

20.Xing M. BRAF mutation in papillary thyroid cancer: pathogenic role, molecular bases, and clinical implications. Endocr Rev 2007; 28: 742-762.

21. Xing M. Prognostic utility of BRAF mutation in papillary thyroid cancer. Mol Cell Endocrinol 2010; 321: 86-93.

22. Horvath E, Majlis S, Rossi R, Franco C, Niedmann JP, Castro A, Dominguez M. An ultrasonogram reporting systems for thyroid nodules stratifying cancer risk for clinical management. J Clin Endocrinol Metab 2009; 94: 1748-1751.

23. Zhang YZ, Xu T, Cui D, Li X, Yao Q. Value of TIRADS, BSRTC and FNA-BRAF V600E mutation analysis in differentiating high-risk thyroid nodules. Sci Rep 2015; 5: 16927.

24. Beisa A, Beisa V, Stoskus M, Ostaneviciute E, Griskevicius L, Strupas K. The value of the repeated examination of BRAF V600E mutation status in diagnostics of papillary thyroid cancer. Endokrynol Pol 2016; 67: 35-40.

25. Gomez Saez JM. Diagnostic and prognostic markers in differentiated thyroid cancer. Curr Genomics 2011; 12: 597-608.

26. Nikiforov YE. Molecular analysis of thyroid tumors. Mod Pathol 2011; 24: 34-43.

27. Kouniavsky G, Zeiger MA. The quest for diagnostic molecular markers for thyroid nodules with indeterminate or suspicious cytology. J Surg Oncol 2012; 105: 438-443.

28. Prasad NB, Kowalski J, Tsai HL. Three-gene molecular diagnostic model for thyroid cancer. Thyroid 2012; 96: 2016-2026.

\section{*Correspondence to}

Shengli-Yan

Endocrinology Department

The Affiliated Hospital of Qingdao University

PR China 